Results: Participants were twenty-two people living with young-onset dementia, fifty-one informal carers and thirty-one formal caregivers. Four themes emerged: (1) impact; (2) coping; (3) service internal functioning; (4) service networking.

The affected people's young age was crucial as it increased the discrepancy between their sentiments of usefulness and inclusion in the society and the attitude of health services and caregivers. Maladaptive coping strategies were put in place as reactions to the diagnosis and in response to the absence of either structures or plans capable of re-uptaking and empowering these people.

Conclusions: Young-onset dementia represents a dramatic turn-of-the-screw in all the people impacted by this diagnosis. One of the needs people living with young-onset diagnosis report relates to social inclusion and maintaining a social role. Considering social needs and enhancing interpersonal interactions and mutual recognition can positively impact individuals' quality of life as it can buffer their limitations and enhance their capabilities.

\title{
302 - The global impact of the COVID-19 pandemic on those living with and caring for someone with dementia
}

Clarissa Giebel, Marta Lenart, Ilaria Chirico, Katarzyna M. Lion

We are presenting four abstracts here for four inter-related talks into the global impact of the pandemic on dementia. Specifically, we will be presenting four talks from our international consortium (UK, India, Italy, Poland, Australia) highlighting how dementia has increased as a global public health concern during the pandemic with similar and different impacts across countries; the mental health impact on people with dementia and carers; the difficulties in using technology for social contact in dementia; as well as the impact on carers with relatives residing in institutional long-term care settings. Public involvement has been a key element of this study. The symposium will be hosted by Dr Clarissa Giebel, the PI of this international 5-country study, and talks will be presented by Dr Giebel, Marta Lenart, Dr Ilaria Chirico, and Dr Katarzyna Lion.

\section{Talk 1 Abstract}

Amplifying dementia as a global public health problem: A cross-country comparison of the impact of COVID-19 pandemic

\section{Clarissa Giebel ${ }^{1,2^{*}}$, Katarzyna Lion ${ }^{3}$, Maria Mackowiak ${ }^{4}$, Rabih Chattat ${ }^{5}$, PN Suresh Kumar ${ }^{6}$, Monica} Cations $^{7}$, Mark Gabbay ${ }^{1,2}$, Wendy Moyle ${ }^{3}$, Giovanni Ottoboni ${ }^{5}$, Joanna Rymaszewska ${ }^{4}$, Adrianna Senczyszyn ${ }^{4}$, Dorota Szczesniak ${ }^{4}$, Hilary Tetlow ${ }^{2}$, Elzbieta Trypka ${ }^{4}$, Marco Valente ${ }^{5}$, Ilaria Chirico ${ }^{5}$

1 Department of Primary Care \& Mental Health, University of Liverpool, UK 2 NIHR ARC NWC, Liverpool, UK

3 Menzies Health Institute Queensland, Griffith University, Australia

4 Department of Psychiatry, Wroclaw Medical University, Poland 5 Department of Psychology, University of Bologna, Italy 6 IQRAA International Hospital and Research Center, Kerala, India 7 College of Education, Psychology and Social Work, Flinders University, Australia

Background: Emerging evidence describes impacts of the COVID-19 pandemic upon people living with dementia and their informal carers, however without evidence-based global comparisons to date. The aim of this international study was to explore and compare the impact of COVID-19 and associated public health restrictions on the lives of people living with dementia and informal carers and access to dementia care across five countries. 